\title{
TLS POINT CLOUD REGISTRATION BASED ON ICP ALGORITHM USING POINT QUALITY
}

\author{
H. Date ${ }^{1 *}$, E. Wakisaka ${ }^{2}$, Y. Moribe ${ }^{3}$, S. Kanai ${ }^{1}$ \\ ${ }^{1}$ Graduate School of Information Science and Technology, Hokkaido University, 060-0814 Sapporo, Japan \\ - (hdate, kanai)@ist.hokudai.ac.jp \\ ${ }^{2}$ Shinryo Corporation, Tsukuba, Japan - wakisaka.ei@shinryo.com \\ ${ }^{3}$ Sanki Engineering Corporation, Kitakyusyu, Japan - moribe@sankieng.co.jp
}

Commission VI, WG VI/4

KEY WORDS: TLS, Point clouds, Registration, ICP Algorithm, weighting, Point quality

\begin{abstract}
:
A simple extension method of the ICP algorithm using point quality based on the distance and incident angle is presented for improving the registration accuracy of TLS point clouds. First, using a structured point cloud representation, the distance and incident angle of each point are calculated. Then, quality measures for the distance and incident angle are defined, considering the relationship between them and the scanning errors. Finally, an extension method of the ICP algorithm by the weighting using the estimated point qualities is described. A method for efficient matching using point properties is also introduced. The proposed method is applied to two datasets of TLS point clouds of a small plant and an outdoor environment, and the effectiveness is evaluated by comparing registration errors with the ground truths obtained using the scan target and a total station.
\end{abstract}

\section{INTRODUCTION}

Registration of TLS (terrestrial laser scanner) point clouds is an initial and critical process for 3D point cloud applications. Because the accuracy of the registration affects the accuracies and reliabilities of applications such as as-built modelling, object recognition, and feature extraction, accurate registration methods are strongly required. For accurate registration of TLS point clouds, scan targets are often set in the environments to be scanned and additional measurements of the target positions using total stations are done. This method can provide reliable registration results. However, the process requires skilled knowledge and experience, and can often be time consuming. Therefore, accurate marker-less automatic registration is strongly required.

The automatic marker-less registration is often done in two steps, i.e. rough and precise (fine) registration. Many methods for rough and precise registration have been proposed (AlDurgham et al., 2013, Al-Durgham et al., 2011, Besl and McKay, 1992, Date et al., 2018, Gressin et al., 2013, Kaneko et al., 2001, Liu et al., 2018, Lohani and Sasidhaan, 2017, Poreba and Goulette, 2015, Rusinkiewicz and Levoy, 2001, Pulli, 1999, Rusu et al., 2009, Sumi et al., 2018, Takai et al., 2013, Theiler et al., 2013, Yang et al., 2016, Yoshimura et al., 2016, Zhu et al., 2016), and several types of scan data can be registered by using appropriate registration methods. Final accuracy of registered point clouds depends on the performance of the precise registration methods, and the ICP algorithm and its variants (Besl and McKay, 1992, Rusinkiewicz and Levoy, 2001) are often used in the precise registration.

The purpose of our research is to improve the registration accuracy for TLS point clouds by the ICP algorithm using the quality of each point in the point clouds. In our research, the distance from the scanner and incident angle of the laser, which affect the measurement errors (Soudarissanane et al., 2011), are used for point quality estimation. In this paper, first, normalized quality measures of each point based on the distance and incident angle are introduced. Then, an extension method of the ICP algorithm considering the point quality is described. Our extension is simply done in the weighting process (Rusinkiewicz and Levoy, 2001), therefore it is quite simple and can be used in several other ICP variants. A method for efficient matching using point properties is also introduced. Finally, experimental results are shown to evaluate the effectiveness of using point quality in the ICP algorithm. The registration errors are compared with the ones of a standard ICP algorithm using the ground truth.

This remainder of this paper is organized as follows: In section 2 , related works are introduced. In section 3, two point quality measures based on the distance and incident angle are described. The extension method of the ICP algorithm using point quality is described in section 4. In section 5, experimental results and quantitative evaluation of the registration accuracy improvement by our method are shown.

\section{RELATED WORK}

Many marker-less automatic registration methods of 3D scan data have been proposed in several area. The registration is generally done in two steps: rough and precise (fine) registration. For precise registration, the ICP algorithm (Besl and McKay, 1992) is often used. The ICP algorithm requires a good initial estimation of positions and orientation for inputs. If a good initial pose and orientation are not provided, incorrect registration results are often obtained, caused by the local

* Corresponding author 
minima of the objective function to be minimized in the ICP algorithm. To obtain correct registration results by the ICP, rough registration is required.

Many rough registration methods for laser-scanned point clouds have been proposed. Among those methods, several features are extracted from input scan data, and they are used to align point clouds. As features, local shape descriptors such as PFH (Rusu et al., 2009), SIFT (Theiler et al., 2013), straight lines (segments) (Al-Durgham et al., 2013, Poreba and Goulette, 2015, Date et al., 2018), 2D feature points (Yang et al., 2016, Yoshimura et al., 2016, Sumi et al., 2018) are used for realizing efficient and robust rough registration. Our focus is precise registration by the ICP algorithm. Therefore, we use roughly aligned point clouds obtained from these methods.

The ICP algorithm (Besl and McKay, 1992) for precise registration iterates finding correspondences (matching) and minimizing an objective function defined by correspondences. Rusinkiewicz and Levoy (Rusinkiewicz and Levoy, 2001) stated that the ICP algorithm consists of sampling, matching, rejection, weighting, and minimization. They compared the registration performances using several options in each step. The framework is used in many following works. For example, Gressin et al. (Gressin et al., 2013) proposed to use several features calculated by PCA in the selection, weighting, and rejection for accurate registration. Al-Durgham et al. (AlDurgham et al., 2011) stated that using projected points on a locally defined surface in the matching produce better registration accuracy. Takai et al. (Takai et al., 2013) showed uniform sampling in the sampling, rejection by dimensionality features in the rejection, and the combined objective functions are useful for accurate registration of MLS point clouds. In our research, we design only the weighting step in the ICP algorithm.

For the weighting, Rusinkiewicz and Levoy (Rusinkiewicz and Levoy, 2001) showed three methods based on the distance between correspondences, difference of normal directions, and uncertainty which represents scan errors estimated from the scan principle. Lie et al. (Liu et al., 2018) also provided a weighting method based on an error model. These researches consider the triangulation-based measurement, and scanner configurations are used for estimating errors. For registration of laser-scanned point clouds, Lohani and Sasidhaan (Lohani and Sasidhaan, 2017) used normalized intensity in the matching to make correspondences more appropriate. Gressin et al. (Gressin et al., 2013) proposed the use of differences of some different features from PCA (eigenvalues, geometrical features, and optimal radii) in the weighting for registration of laser scanned point clouds of large-scale environments. For robust registration against to scanning noise and outliers, Kaneko et al. (Kaneko et al., 2001) introduced M-Estimator for the weighting. In our research, we propose a weighting method based on the quality of laser-scanned point using the measurement distance and incident angle.

Point quality evaluation of point clouds obtained from laserscanning has been conducted (Soudarissanane et al., 2009, Soudarissanane et al., 2011, Ozendi et al., 2017, Wujanz et al. 2017). It is reported that measurement distance, incident angles

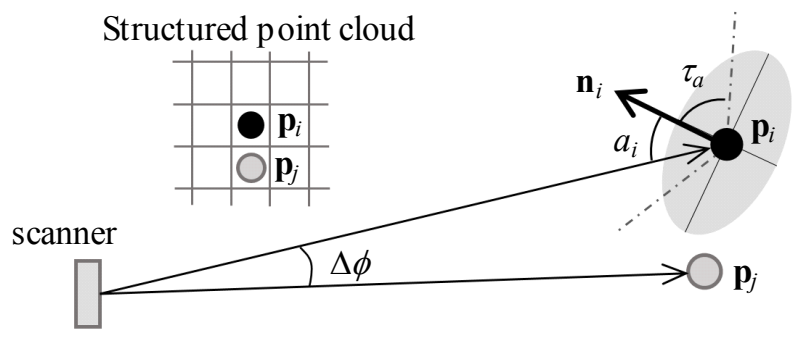

Figure 1. Neighbor point identification and incident angle

of the laser, and reflectance intensity of the laser are important factors for precision and accuracy of scanned points, and their relationships are investigated. Soudarissanane et al. (Soudarissanane et al., 2009, Soudarissanane et al., 2011) summarized the main factors influencing quality of each point from laser scanning, and evaluated the influence of the distance and incident angles for the measurement errors. Stochastics and generic error models of points from TLS have been developed based on the measurement principles and experiments (Ozendi et al., 2017, Wujanz et al. 2017). In our research, we consider the distance and incident angle to evaluate the quality of each point in laser-scanned point clouds. Simple scalar measures for point quality are defined based on the results of previous works (Soudarissanane et al., 2009, Soudarissanane et al., 2011), and used in the ICP algorithm.

\section{POINT QUALITY MEASURE}

Precision and accuracy of laser-scanned point clouds depend on several factors (Soudarissanane et al., 2011), such as distance from the scanner, incident angle of the laser, surface properties of the object, atmospheric conditions, and shape of the laser spot. In each point from laser scanning, coordinates and laser intensity can be obtained by most TLS systems. Although the intensity can be considered as one of the point qualities, we do not use the intensity in point quality evaluation because the intensity is affected by various factors and some systems often produce unreliable intensity information. In our research, distance from the scanner and incident angles, which can be calculated by the coordinates, are used.

Generally, the origin of the TLS point clouds is the scanner position. Therefore, the distance of each point $i$ from scanner, $d i$, is obtained as the norm of the position vector of point $i$, i.e. $d_{i}=\left\|\mathbf{p}_{i}\right\|$, where $\mathbf{p}_{i}$ is the position vector of the point $i$.

To calculate the incident angle of each point $i, a_{i}$, first, a normal vector at the point $i, \mathbf{n}_{i}$, is estimated. We calculate the normal vector as the average of the outer products of the difference vectors for its neighboring points. To find appropriate neighbors efficiently, structured representation of points (2D grid representation on azimuth and horizontal angle plane (Masuda and Tanaka, 2010)) is used. For each point on the grid representation, neighbors of a point $i$ are defined by Eq. (1) using plane approximation around point $i$ (Chiba and Masuda, 2016), as shown in Figure 1. 


$$
i^{*}=\left\{j \in N(i) \mid\left\|\mathbf{p}_{i}-\mathbf{p}_{j}\right\|<\frac{\left(\left\|\mathbf{p}_{i}\right\|+\left\|\mathbf{p}_{j}\right\|\right) \Delta \phi}{2 \cos \tau_{a}}\right\}
$$

where $N(i)=$ four neighbor points of point $i$ on the structured point clouds

$\Delta \phi=$ angle pitch in the scanning

$\tau_{a}=$ upper bound of acceptable incident angle

Using the neighbors, the unit normal vector $\mathbf{n}_{i}$ is estimated as an average vector of outer products of difference vectors between point $i$ and its neighbors. The incident angle $a_{i}$ is estimated by $a_{i}=-\arccos \left(\mathbf{n}_{i} \cdot \mathbf{p}_{i} / d_{i}\right)$.

\section{Distance quality measure}

It is reported that the precision of a laser scan becomes higher as the distance of the scanned surface from the scanner increases until a certain distance, and if the distance becomes larger than that certain distance, the precision becomes smaller depending on the distance (Soudarissanane et al., 2011). Although the value of the precision (e.g. standard deviation) depends on the scanning system, the tendency of the precision and the distance is often common. According to the results of the investigation of the laser scanning precision, the precision can be roughly approximated by two quadratic functions of the distance before and after the certain distance. Therefore, in our research, we define a normalized distance quality measure of each point $i, q_{i}^{D S T}$ by a combination of quadratic functions by Eq. (2).

$$
q_{i}^{D S T}= \begin{cases}1-\left(1-q_{0}\right)\left(d_{i}-d_{c}\right)^{2} / d_{c}^{2} & \left(d_{i}<d_{c}\right) \\ 1-\left(d_{i}-d_{c}\right)^{2} /\left(d_{m}-d_{c}\right)^{2} & \left(d_{c} \leq d_{i}<d_{m}\right) \\ 0 & \text { otherwise }\end{cases}
$$

where $\quad d_{c}, d_{m}, q_{0}=$ parameters

The function is illustrated in Fig. 2(a). Ideally, the parameters should be determined according to the property of the scanner used. The discussion of the parameter setting in our experiments is described in section 5 .

\section{Angle quality measure}

The measurement accuracy drastically decreases if the incident angle becomes larger than a certain incident angle (Soudarissanane et al., 2011). It is known that the deterioration of a reflected laser and the accuracy of measurement follows $c / \cos \theta$, where $\theta$ is the incident angle of the laser and $c$ is a coefficient. Therefore, the normalized inverse of $m-c / \cos \theta$, where $m$ is the maximum degree of deterioration decided by the acceptable maximum incident angle, can be used as the quality of points related to the incident angle. As a result, we can approximate the angle quality of each point $i, q_{i}^{A N G}$, using Eq. (3).

$$
q_{i}^{A N G}= \begin{cases}\frac{1-\cos \tau_{a} / \cos a_{i}}{1-\cos \tau_{a}} & \left(a_{i}<\tau_{a}\right) \\ 0 & \text { otherwise }\end{cases}
$$

where $\quad \tau_{a}=$ acceptable maximum incident angle

The function is shown in Fig. 2(b). The parameter $\tau_{a}$ decides the degree of attenuation of the angle quality and acceptable

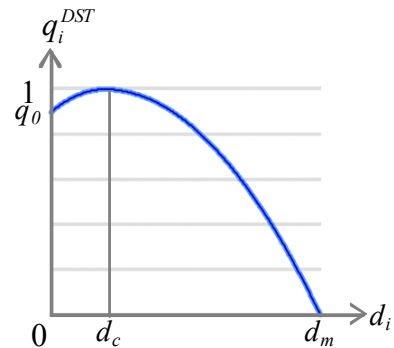

(a) Distance quality

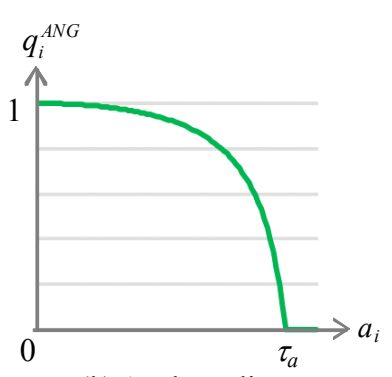

(b) Angle quality
Figure 2. Point quality measures

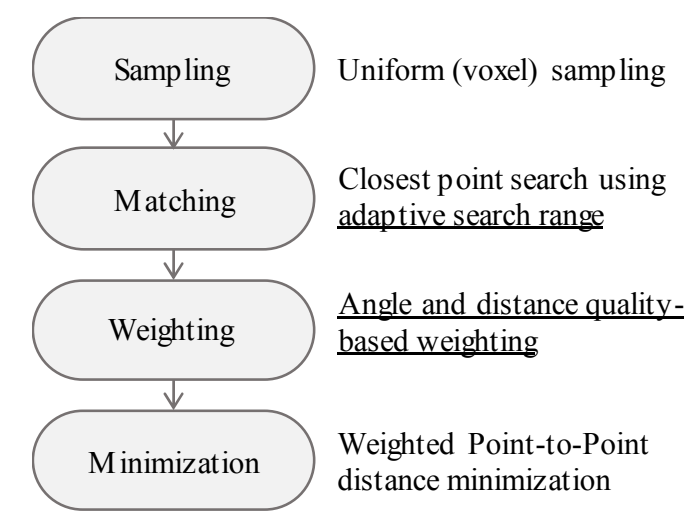

Figure 3. ICP Algorithm used in our research

maximum incident angle. Determination of the $\tau_{a}$ in our experiments is also described in section 5 .

\section{ICP ALGORITHM USING POINT QUALITY}

Basic components of the ICP algorithm were summarized in a previous study (Rusinkiewicz and Levoy, 2001). The algorithm consists of sampling, matching, rejection, weighting and minimization. In our method, we design the weighting process based on the point quality. Figure 3 shows the algorithm used in our research. In sampling, we use uniform (voxel) sampling, and matching is done by closest point search using a kd-tree. In the matching process, a simple method for determining the appropriate search range based on the basic point properties is introduced for efficient computation. The qualities defined by Eqs. (2) and (3) are used in the weighting, and an objective function defined by Eq. (4) is used in the minimization.

$$
\sum_{(i, j) \in C} w_{i j}\left\|\mathbf{q}_{j}-\left(\mathbf{R} \mathbf{p}_{i}+\mathbf{t}\right)\right\|^{2}
$$

where $\quad C=$ a set of corresponding point pairs $\{(i, j)\}$

$\mathbf{p}_{i}=$ position of point $i$ in the movable point cloud

$\mathbf{q}_{j}=$ position of point $j$ in the fixed point cloud

$\mathbf{R}=\left\{r_{i j}\right\}=3 \times 3$ rotation matrix

$\mathbf{t}=$ translation vector

$w_{i j}=$ weight for point pair $(i, j)$

Minimizing Eq.(4) is to find the rotation matrix $\mathbf{R}$ and the translation vector $\mathbf{t}$ which minimize the sum of squared distances between the closest points. Many researches indicated that the minimizing point-to-plane distances can realize more accurate registration than point-to-point distance minimization (Rusinkiewicz and Levoy, 2001). However, point-to-plane minimization requires appropriate normals, and it is often difficult to apply the method to several environments (Takai et 
al., 2013). To remove instable factors in the evaluation of the influence of the point quality, we use point-to-point minimization in our experiments. In the remaining part of this section, a search range determination method based on the basic point properties for efficient matching, and the weighting method based on the point quality are described.

\section{Adaptive search range in matching}

Closest point search using an appropriate upper search range can reduce inappropriate correspondences and realize efficient neighbor search. If a candidate corresponding point $j$ for a query point $i$ is found, using similar idea to Eq.(1), the distance between neighbor points around the point $j$ can be estimated by Eq.(5).

$$
s_{j}=d_{j} \times \Delta \phi / \cos a_{j}
$$

Therefore, $s_{j}$ can be used for a search range if the proper corresponding point exists near point $j$. In the ICP algorithm, under good initial pose estimation, fine correspondences can be found in the early stage of the iteration. In our method, after a few iterations using a large search range, the scaled $s_{j}$ is used for the sampled point $i$ corresponding to point $j$ as the search range. Finally, a small search range is used for precise matching.

\section{Weighting}

The weight $w_{i j}$ in Eq. (4) can control the degree of importance of corresponding point pair $(i, j)$ in the minimization for deriving coordinate transformation. Several options for determining the weight for each point pair can be considered. In our research, we assumed that the worst quality is dominant for the registration accuracy. Therefore, by using Eq. (6), a minimum quality for the point pair $(i, j)$ is used as its weight $w_{i j}$.

$$
w_{i j}=\min \left(q_{i}^{D S T}, q_{i}^{A N G}, q_{j}^{D S T}, q_{j}^{A N G}\right)
$$

The quality measures can be used for the rejection in order to reject extremely unreliable pairs with inappropriate points, such as points at vary high incident angles. In our method, this can be achieved by weighting using the quality measures with a value of 0 for inappropriate points. The quality measures of each point can be pre-computed and are unchanged in the ICP algorithm. Therefore, computational cost of the ICP algorithm does not change by this weighting.

\section{EXPERIMENTAL RESULTS}

\subsection{Datasets and method for evaluation}

Table 1 summarizes two datasets used in our experiments, and Figures 4 and 5 show the colored point clouds and scan positions of each dataset. Dataset A is a set of point clouds acquired from a small heat source plant, and Dataset B is from outdoor environments including buildings. The point clouds were acquired by a TLS (Z+F IMAGER 5010C). Coordinate transformation matrices and vectors of the ground truth (ideal registration) for error evaluation were obtained by measurement using a total station (Leica TCRP 1205+) and targets. The center positions of the planar targets colored by black and white are calculated from the point clouds and their positions are aligned to the reference coordinates measured by the total station.

\begin{tabular}{|c|c|c|}
\hline & Dataset A & Dataset B \\
\hline Scan site & Heat Source Plant & Outdoor \\
\hline \#scans & 10 & 3 \\
\hline \#points/scan & $42.9 \mathrm{M}-43.4 \mathrm{M}$ & $29.0 \mathrm{M}-29.9 \mathrm{M}$ \\
\hline
\end{tabular}

Table 1. Datasets used in the experiments
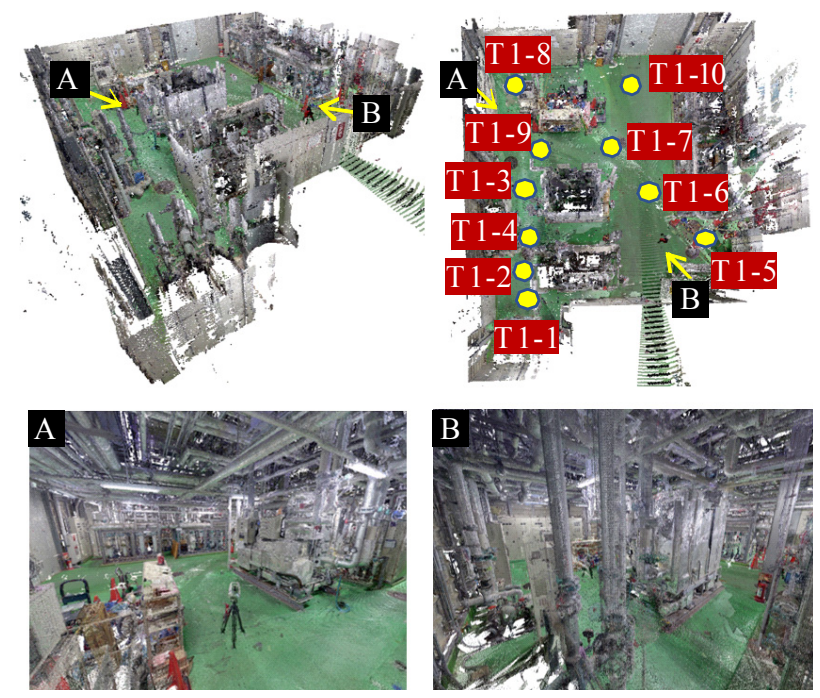

Figure 4. Point clouds of Dataset A
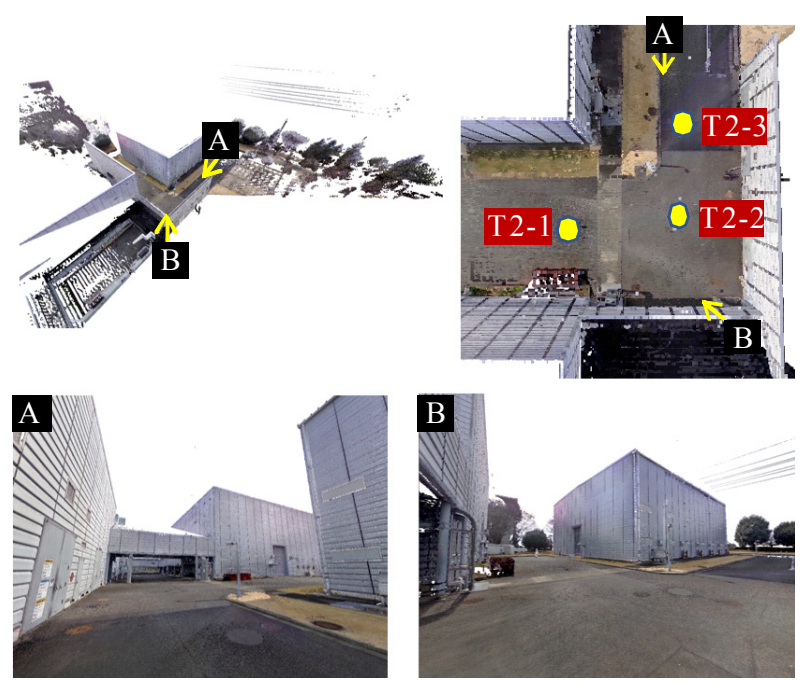

Figure 5. Point clouds of Dataset B

The translation and rotation errors after the registration by the ICP algorithm are calculated by using rotation matrices and translation vectors of the ground truth and from the ICP algorithm using Eqs. (7) and (8).

$$
\begin{aligned}
& e_{T}=\left\|\mathbf{t}^{\prime}-\mathbf{t}\right\| \\
& e_{R}=\sum_{i=1}^{3} \sum_{j=i}^{3}\left|r_{i j}^{\prime}-r_{i j}\right|
\end{aligned}
$$

where $\quad \mathbf{t}^{\prime}=$ translation vector of the ideal registration $\mathbf{R}^{\prime}=\left\{r_{i j}^{\prime}\right\}=3 \times 3$ rotation matrix of the ideal registration 


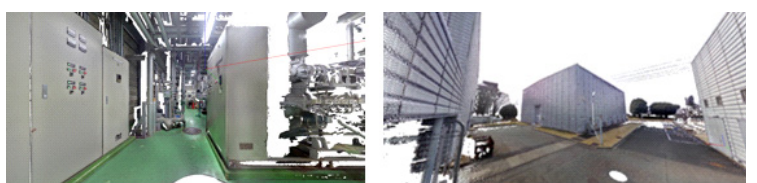

(a) Point clouds
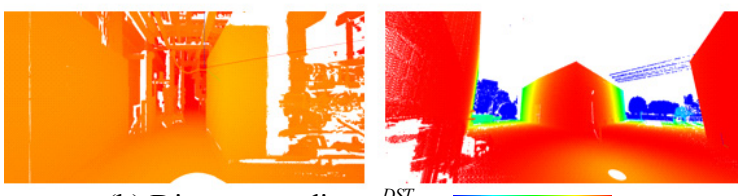

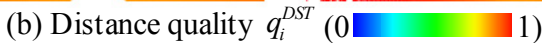
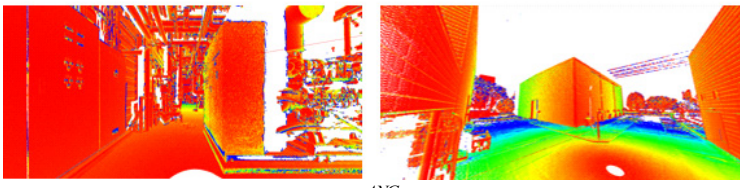

(c) Angle quality $q_{i}^{A N G}(0 \square 1)$

Figure 6. Point Quality

To evaluate the effectiveness of our method, errors are calculated for registration results of the ICP algorithms with constant weights and our weighted versions, and they are compared. Evaluations are done for some pairwise registration results for neighboring point clouds.

The performance of weighting depends on the parameters of quality measures in Eqs. (2) and (3). A best method for determination of optimal parameters is to fit the functions to the precision and accuracy evaluation results using the scanner for acquiring the point clouds. On the other hand, in our research, we consider that the exact quality values are not necessarily required because the quality values are used only in weighting. If the tendency of the quality measures fit the tendency of the precision and accuracy of laser scanning, the registration results will be improved. The degree of improvement may be optimized by using the best parameter decision method. In our experiments, we set the parameters according to the reported results in the related works (Soudarissanane et al., 2009, Soudarissanane et al., 2011) and our measurement experiments. The precision becomes the highest at $10 \mathrm{~m}$ from the scanners. An incident angle larger than approximately 60 70 degrees results in inaccurate points. According to these observations, in our experiments, $10 \mathrm{~m}$ and $85 \mathrm{deg}$ are used for $d_{c}$ and $\tau_{a}$, respectively. Other parameters for distance quality are decided experimentally. $d_{m}=50 \mathrm{~m}$ and $q_{0}=0.8$ are used in the experiments. Figure 6 shows the distance and angle qualities calculated using these parameters.

\subsection{Experimental results and evaluations}

Figures 7 shows the registration errors defined in Eqs. (7) and (8) for two datasets. In each graph, the errors from uniform weight $\left(w_{i j}=1\right)$ and proposed quality-based weight are shown. In the figure, (a) and (b) show the translation and rotation errors respectively, and (c) represents the pair ID and corresponding scanner positions in each dataset (Figures 4 and 5).

From the experimental results, it was observed that the qualitybased weight can reduce translation and rotation errors for almost all point cloud pairs. Average improvement rates of the translation and rotation errors were $6.0 \%$ and $1.0 \%$ for Dataset $\mathrm{A}$, and $6.0 \%$ and $3.7 \%$ for Dataset B. Maximum improvement

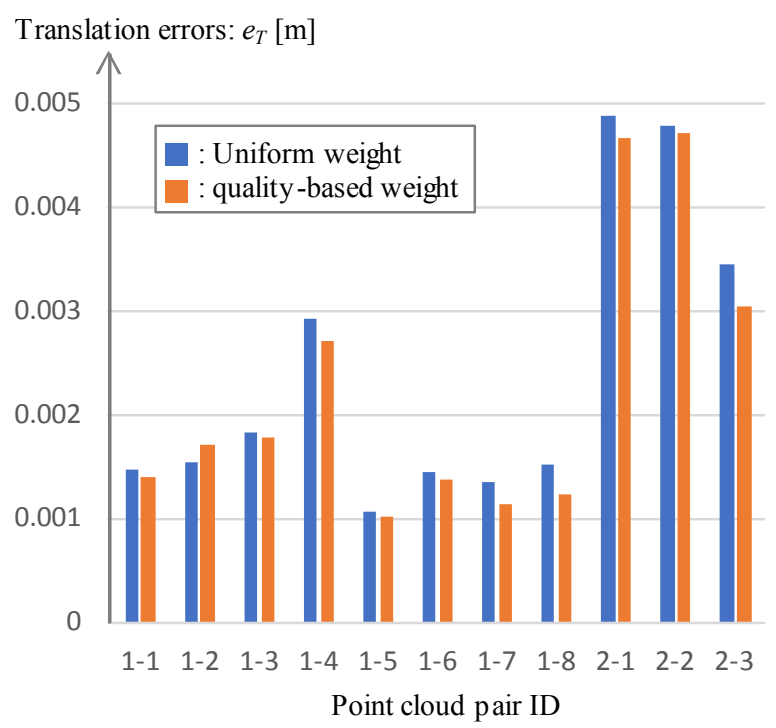

(a) Translation errors: $e_{T}$

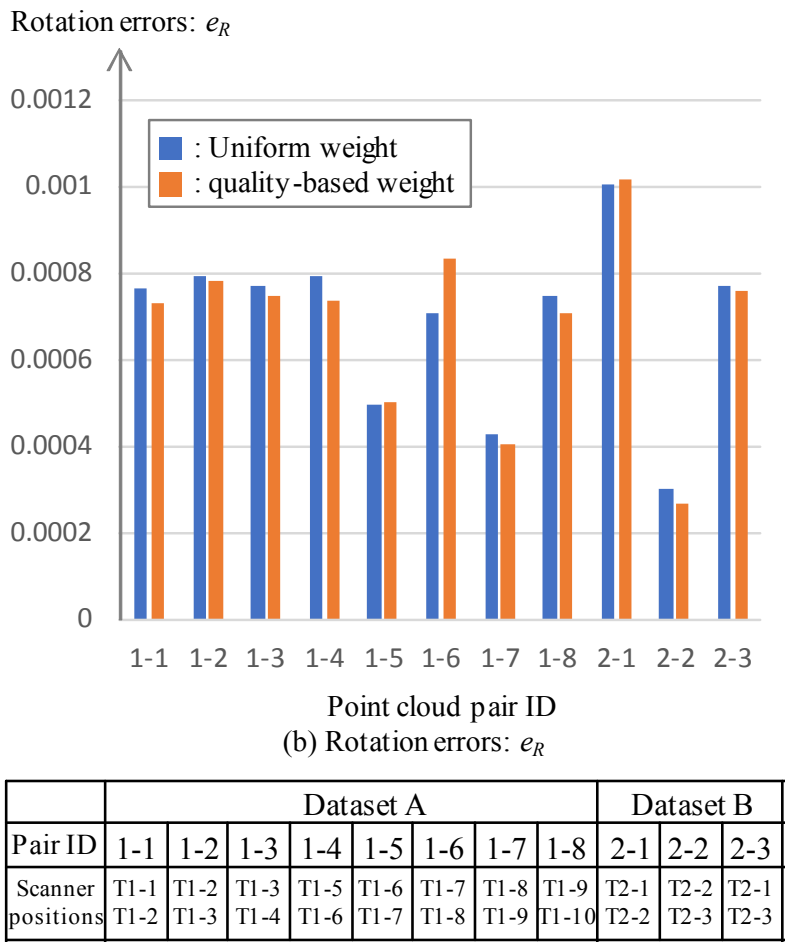

(c) Pair ID and corresponding scanner positions

Figure 7. Registration errors

rates of the translation and rotation errors were $18.7 \%$ and $10.8 \%$, respectively. The errors were reduced in almost all cases, on the other hand, in some results, the errors increased (translation: 1/11, rotation: 3/11). Detailed analysis of the property of the point clouds which results in larger errors will be included in future work. The quality of the point clouds used in the experiments can be considered relatively high because a relatively high quality scanner is used experiments. Nevertheless, a clear tendency of improvement in the registration accuracy was observed. More experiments for several environments and scanners will also be included in future work. 
We implemented a kd-tree for closest point search in the matching. By using an adaptive search distance, computation times for iterations in the ICP algorithm were reduced to about $20 \sim 30 \%$.

\section{CONCLUSIONS}

In this paper, an extension method of the ICP algorithm based on point quality was introduced to realize more accurate registration of TLS point clouds. First, the point quality measures based on the distance from the scanner and incident angle of the laser were introduced. Then, a weighting method using point quality measures in the ICP algorithm is proposed. Experimental results using two datasets from indoor and outdoor environments showed that the weighting by point quality can improve the registration accuracy. The maximum improvement rates of the translation and rotation errors were $18.7 \%$ and $10.8 \%$ in the experiments. Additional experiments for different environments and scanners, and detailed analysis for error increasing cases will be included in future works.

\section{ACKNOWLEDGEMENT}

This work was supported by JSPS KAKENHI Grant Number 18K03896.

\section{REFERENCES}

Al-Durgham, K., Habib, A., Kwak, E., 2013. RANSAC Approach for Automated Registration of Terrestrial Laser Scans using Linear Features. ISPRS Ann. Photogramm. Remote Sens. Spatial Inf. Sci., II-5/W2, 13-18.

Al-Durgham, M., Detchev, I., Habib, A., 2011. Analysis of two triangle-based multi-surface registration algorithms of irregular point clouds. Int. Arch. Photogramm. Remote Sens. Spatial Inf. Sci., XXXVIII-5/W12, 61-66.

Besl, P. J. and McKay, N. D., 1992. A Method for Registration of 3D Shapes. IEEE Transactions on Pattern Analysis and Machine Intelligence, 14(2), 239-256.

Chiba, A. and Masuda, H., 2016. Reconstruction of polygonal prisms from point-clouds of engineering facilities. Journal of Computational Design and Engineering, 3, 322-329.

Date, H., Yokoyama, T., Kanai, S., Hada, Y., Nakao, M, Sugawara, T., 2018. Efficient Registration of Laser-Scanned Point Clouds of Bridges Using Linear Features. International Journal of Automation Technology, 12(3), 328-338.

Gressin, A., Mallet, C. Demantké, J., David., A., 2013. Towards 3D lidar point cloud registration improvement using optimal neighborhood knowledge. ISPRS Journal of Photogrammetry and Remote Sensing, 79, 240-251.

Kaneko, S., Kondo, T., Miyamoto, A., Igarashi, S., 2001. Robust ICP Registration Algorithm Extended by M-estimation. Journal of the Japan Society of Precision Engineering, 67(8), 1276-1280.

Liu, S., Gao, D., Wang, P. Guo, X., Xu, J., Liu D.X., 2018. A Depth-Based Weighted point Cloud Registration for Indoor Scene. Sensors, 18, 3608.
Lohani, B. and Sasidhaan, S., 2017. An evaluation of intensity augmented ICP for terrestrial LiDAR data registration. Journal of Geomatics, 11(2), 139-148.

Masuda, H. and Tanaka, I., 2010. As-Built 3D Modeling of Large Facilities Based on Interactive Feature Editing. Computer-Aided Design and Applications, 7(3), 349-360.

Ozendi, M., Akca, D., Topan, H., 2017. A generic point error model for TLS derived point clouds. In: Proceedings of SPIE, 10332, 103320J.

Poreba, M. and Goulette F., 2015. A Robust Linear FeatureBased Procedure for Automated Registration of Point Clouds, Sensors, 15, 1435-1457.

Rusinkiewicz, S. and Levoy, M., 2001. Efficient Variants of the ICP Algorithm. In: Proceedings of Third International Conference on 3-D Digital Imaging and Modeling, 145-152.

Rusu, R. B., Blodow, N., Beetz, M., 2009. Fast point feature histograms (FPFH) for 3d registration. In: Proceedings of IEEE International Conference on Robotics and Automation, 32123217 .

Soudarissanane, S., Lindenbergh, R., Menenti, M., Teunissen, P.J.G., 2009. Incidence angle influence on the quality of terrestrial laser scanning points. In: Proceedings of ISPRS Workshop Laser scanning 2009, 183-188.

Soudarissanane, S., Lindenbergh, R., Menenti, M., Teunissen, P., 2011. Scanning geometry: Influencing factor on the quality of terrestrial laser scanning points. ISPRS Journal of Photogrammetry and Remote Sensing, 66, 389-399.

Sumi, T., Date, H., Kanai, S., 2018. Multiple TLS point cloud registration based on point projection images. Int. Arch. Photogramm. Remote Sens. Spatial Inf. Sci., XLII-2, 1083-1090.

Takai, S., Date, H., Kanai, S., Niina, Y., Oda, K., Ikeda, T., 2013. Accurate registration of MMS point clouds of urban areas using trajectory. ISPRS Ann. Photogramm. Remote Sens. Spatial Inf. Sci., II-5/W2, 277-282.

Theiler, P. W., Wegner, J. D., Schindler, K., 2013. Markerless point cloud registration with keypoint-based 4-points congruent sets. ISPRS Ann. Photogramm. Remote Sens. Spatial Inf. Sci., II-5/W2, pp. 283-288.

Wujanz, D., Burger, M., Mettenleiter, M., Neitzel, F., 2017. An intensity-based stochastic model for terrestrial laser scanners. ISPRS Journal of Photogrammetry and Remote Sensing, 125, 146-155.

Yang, B., Dong, Z., Liang, F., Liu, Y., 2016. Automatic registration of large-scale urban scene point clouds based on semantic feature points. ISPRS Journal of Photogrammetry and Remote Sensing, 113, 43-58.

Yoshimura, R., Date, H., Kanai, S., Honma, R., Oda, K., Ikeda, T., 2016. Automatic Registration of MLS Point Clouds and SfM Meshes of Urban Area. Geo-spatial Information Science, 19(3), 171-181. 